山्山FANÇAISE

$>\mathrm{DE}$

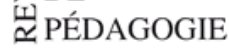

\section{Revue française de pédagogie}

Recherches en éducation

171 | avril-juin 2010

La mixité scolaire : une thématique (encore)

d'actualité ?

\title{
ROUILLER Yviane \& LEHRAUS Katia (dir.). Vers des apprentissages en coopération : rencontres et perspectives
}

Berne : Peter Lang, 2008, 237 p.

Christine Brisset

\section{OpenEdition}

Journals

Édition électronique

URL : http://journals.openedition.org/rfp/1974

DOI : $10.4000 / \mathrm{rfp} .1974$

ISSN : 2105-2913

Éditeur

ENS Éditions

Édition imprimée

Date de publication : 1 juillet 2010

Pagination : 158-160

ISBN : 978-2-7342-1187-7

ISSN : 0556-7807

Référence électronique

Christine Brisset, «Roulluer Yviane \& Lehraus Katia (dir.). Vers des apprentissages en coopération : rencontres et perspectives », Revue française de pédagogie [En ligne], 171 | avril-juin 2010, mis en ligne le 12 octobre 2010, consulté le 24 septembre 2020. URL : http://journals.openedition.org/rfp/1974 ; DOI : https://doi.org/10.4000/rfp.1974

Ce document a été généré automatiquement le 24 septembre 2020.

(c) tous droits réservés 


\title{
ROUILLER Yviane \& LEHRAUS Katia (dir.). Vers des apprentissages en coopération : rencontres et perspectives
}

Berne : Peter Lang, 2008, 237 p.

\author{
Christine Brisset
}

\section{RÉFÉRENCE}

ROUILLER Yviane \& LEHRAUS Katia (dir.). Vers des apprentissages en coopération : rencontres et perspectives. Berne : Peter Lang, 2008, 237 p.

1 La vocation de cette collection (Exploration. Recherches en sciences de l'éducation de la société suisse pour la recherche en éducation), publiée sous la direction de Marcel Crahay, Rita Hofstetter, Bernard Schneuwly et Maurice Tardif, est de mettre en relief la pluralité des sciences de l'éducation comme « carrefour des multiples dimensions de la recherche et de l'action éducative ". Ce livre est la synthèse de réflexions ayant leur origine dans différents colloques du Réseau éducation et formation, dont celui de Genève en septembre 2003. Les deux coordonnatrices de ce livre ont d'abord été enseignantes dans le premier degré puis formatrices. Yviane Rouiller travaille actuellement à la Haute école pédagogique du canton de Vaud. Katia Lehraus est chargée d'enseignement à la faculté de psychologie et de sciences de l'éducation de Genève. Elles ont pris un risque certain en présentant un ouvrage sur un sujet aussi diversifié et actuel que l'impact des interactions dans l'apprentissage. Et c'est certainement une des forces de ce livre: des références d'auteurs multiples et pertinentes, des approches diversifiées, une discussion dynamique. C'est ce qui en constitue cependant parfois aussi les faiblesses car les quinze contributeurs ont une pluralité d'approches qui entraîne aussi une difficulté de centration sur le choix de l'objet d'étude défini dès le titre : les apprentissages en coopération. En effet les auteurs vont éclairer, chacun à leur manière et selon leur spécialité, les différentes modalités 
d'interactions que sont donc la coopération mais aussi la collaboration et le «travail en groupe ». Un bémol est apporté par Tardif lui-même, dès la préface, concernant la mise en perspective des deux derniers. Ce livre, nous dit-il, «ne fournit pas les données nécessaires pour empêcher que la collaboration et le travail en groupe soient examinés d'une manière simplificatrice ou "expansionniste" " (p. X). Et nous ressentons souvent au fil de la lecture une oscillation entre le désir, d'une part, de bien distinguer les différentes possibilités de travail à plusieurs et donc d'en exposer les principales modalités en les comparant et, d'autre part, de conserver une réflexion commune centrée sur la pédagogie coopérative. Dans cet essai de définition de la notion d'«interactions planifiées entre pairs", il manque parfois ce que Bruner appelle «l'attention conjointe ». C'est sans doute, en partageant encore plus l'objectif défini de s'intéresser à un type de pédagogie, que les contributeurs auraient pu parfaire l'élaboration d'une signification commune. Mais cet ouvrage offre une analyse très dynamique et référencée de ce qu'est et de ce que devrait être la pédagogie coopérative.

2 Dès le début de cet ouvrage, Jacques Tardif met en avant une question centrale: "Quelles sont les caractéristiques et les composantes des interactions sociales entre des élèves ou des étudiants qui contribuent à des apprentissages signifiants? » (p. IX) Dans l'introduction, Lehraus et Rouiller exposent deux ancrages théoriques fondateurs des approches coopératives: d'une part, les travaux de Piaget réactualisés par l'école de Genève et, d'autre part, ceux de Vygotski avec les compléments effectués par exemple par Schneuwly ou Rivière. Deux ouvertures sur le terrain scolaire vont venir compléter cette perspective théorique: l'une provenant de l'Europe francophone mettant en avant les grands noms et idées fortes des mouvements de l'« Éducation nouvelle » et des réflexions plus récentes comme celles de Nicolet, Carbonnel, Meirieu, Pléty, Baudrit; la seconde ouverture nous amène en pays nord-américain, avec par exemple une mise en avant des travaux de Dewey mais aussi de Deutsch, de Johnson et Johnson, de Palincsar et Brown, etc. Après la préface et l'introduction, huit chapitres divisés en deux grandes parties offrent un panorama de recherches et de réflexions clôturé par un chapitre concernant plus spécifiquement la formation des enseignants et enfin par un essai de «modélisation des conditions favorables aux apprentissages coopératifs ».

3 La première partie (137 pages) comprend cinq contributions dont la clôture de Marcel Crahay. Pour commencer, Nuno Bessa et Anne-Marie Fontaine, tous deux enseignants au Portugal, font une présentation d'une expérimentation faite en lycée professionnel ; il s'agit de regarder les effets de la méthode STAD (Student teams achievement divisions), méthode coopérative développée aux États-Unis par Robert Slavin et visant à améliorer la réussite scolaire et la motivation. Les résultats (en décalage avec ceux de Slavin, 1996) d'une recherche développée au Portugal sont décrits et analysés, des pistes d'action sont proposées. Dans le chapitre suivant, c'est une des élèves de Butera et de Mugny, Céline Buchs (enseignant-chercheur à l'université de Genève), qui présente un ensemble d'études afin « d'examiner dans quelles conditions et par quels processus les dispositifs d'apprentissage entre pairs sont les plus efficaces» (p.58), ceci au sein du travail coopératif lors des travaux dirigés à l'université, afin de proposer une pédagogie plus adaptée. Le même type de contribution suit avec l'exposé par Yviane Rouiller d'une revue de littérature et de résultats mettant en valeur les avantages des interactions entre élèves lors de productions écrites. L'auteur va alors présenter des études concernant des élèves de dix à douze ans mis en situation de production 
coopérative soit de textes narratifs, soit de révision de textes, en se centrant plus particulièrement sur la composition des équipes concernant le genre des élèves et leur niveau de connaissances. La conclusion insiste sur la nécessité mais aussi la difficulté de transposition des recherches dans des situations réelles de classe. Et c'est sur cette dernière question que rebondit la contribution de Michael Baker, chercheur au CNRS de Paris. Il analyse en effet le décalage souvent observé entre les savoirs théoriques et les pratiques éducatives. S'appuyant entre autres sur les travaux de Gilly, Roux, Trognon, Crahay et sur les résultats de ses propres recherches, Baker va s'interroger sur la nature des savoirs et sur les moyens et les pratiques de l'activité coopérative. Pour réunifier les différents axes et niveaux d'analyse, Marcel Crahay clôt ce chapitre en proposant d'édifier un cadre conceptuel « des conditions d'efficacité de la pédagogie coopérative». Tout en citant un travail comparable (celui de Webb et Palincsar en 1996), il s'en démarque dans la manière de procéder. Ainsi, pour chacune des analyses, il va en extraire la caractéristique permettant de construire ce modèle conceptuel. Par ce travail de recentrage, il énonce de façon plus homogène les conditions d'efficacité de la pédagogie coopérative.

La seconde partie est beaucoup plus courte ( 52 pages) et se termine par un contrepoint de nouveau fort appréciable. Le premier chapitre rédigé par des chercheurs belges (Benoît Galand, Étienne Bourgeois, Mariane Frenay et Kathleen Bentein) est l'exposé d'une réflexion comparative entre l'apprentissage coopératif et l'apprentissage par problème (APP), "une méthode pédagogique qui connaît un succès croissant dans l'enseignement supérieur » (p. 140). En présentant différentes recherches (dont celles de Pochet, Dochy, Segers, Johnson et Johnson), les auteurs interrogent les conditions d'efficacité de ces deux types de travail interactif et présentent une étude portant sur les perceptions des étudiants envers les pratiques pédagogiques auxquelles ils sont confrontés. Après un exposé des résultats d'une étude faite auprès de plus de 800 étudiants de première et troisième années en formation d'ingénieur, des liens sont opérés avec d'autres études. Le deuxième chapitre sera totalement différent ; MarieAnne Hugon, professeur de sciences de l'éducation, membre du Centre de recherche en éducation et en formation de l'université Paris Ouest-Nanterre-La Défense et ancienne chercheur au CRESAS (INRP), a axé son écriture sur l'exposé des axes principaux de ce groupe aujourd'hui disparu. Après en avoir retracé le cadre théorique, Hugon expose plus particulièrement deux recherches menées au collège et au lycée qui vont lui permettre d'expliciter les caractéristiques déterminantes pour l'efficacité d'une pédagogie coopérative. Le contrepoint de Jean-Marie De Ketele arrive à point nommé pour développer des ponts entre ces deux contributions. Par une réelle distance critique, il en analyse les principaux apports et inconvénients. Un dernier chapitre s'intéresse à un sujet abordé par quelques auteurs précédents : celui de la transposition de résultats de recherche sur la coopération aux pratiques de classe. Katia Lehraus, une des deux coordonnatrices, et Martine Sabourin (dont on n'a pas la présentation mais dont on connaît l'ampleur des travaux sur la coopération) vont mettre en avant l'impact de deux dispositifs de formation continue des enseignants, l'un à Montréal, l'autre à Genève. Une revue de littérature énumère les conditions d'efficacité d'une formation et l'analyse des résultats aboutit à un questionnement élargi sur la politique d'un pays. Or cette question nous apparaît ô combien centrale dans le contexte actuel français de la réforme de la formation des enseignants.

5 La conclusion tente de resserrer l'analyse très éclectique des différentes contributions. Rouiller et Lehraus rappellent l'ampleur des différences d'appellations, de définitions, 
de buts... et leur envie de faire le point sur cette diversité. On comprend bien en quoi la cohérence d'un tel ouvrage était compliquée à développer. Elles reprennent ici la discussion qu'elles avaient amorcée en introduction afin de rappeler ce que chaque auteur a pu amener au débat. En élaborant un cadre conceptuel fait des apports de chacun, elles témoignent à leur manière d'un essai de coopération qui pourrait aller audelà des études relatées. C'est donc pourquoi, mis à part quelques bémols précédemment énoncés, cet ouvrage apporte sa pierre à l'édifice socioconstructiviste. Des corpus d'élèves, des tableaux synthétiques, des schémas, des encadrés viennent souvent clarifier les propos. Sont également présentes de riches bibliographies et les citations d'auteurs au sein des textes proposés permettent d'avoir au fil de la lecture les travaux de base structurant les recherches à ce sujet. Ce livre constitue donc un bon support pour des étudiants en recherche d'informations sur le sujet ou des formateurs voulant réfléchir et/ou mettre en pratique une pédagogie coopérative. De par la diversité des pistes proposées, il pourrait également constituer un point d'ancrage à l'élaboration de nouvelles recherches.

\section{AUTEURS}

CHRISTINE BRISSET

IUFM d'Amiens et laboratoire du CLEA, université de Picardie-Jules-Verne 